\title{
Primary intramedullary melanocytoma in the thoracic cord: a case report and literature review
}

\author{
Chao Wang ${ }^{1}$, Xiaotong Shao ${ }^{1}$, Yi Zou ${ }^{2}$ \\ ${ }^{1}$ Department of Radiology, the Second Affiliated Hospital, Zhejiang University School of Medicine, Hangzhou, China; ${ }^{2}$ Department of Pathology, \\ the Second Affiliated Hospital, Zhejiang University School of Medicine, Hangzhou, China \\ Correspondence to: Dr. Chao Wang. Department of Radiology, the Second Affiliated Hospital, Zhejiang University School of Medicine, No.88 Jiefang \\ Road, Hangzhou 310009, China. Email: wangchaosmart@zju.edu.cn.
}

\begin{abstract}
Background: Primary intramedullary melanocytoma (PIM) is extremely rare, only 25 cases of PIM have been reported previously. Herein we report a case of PIM in the thoracic cord and reviewed its clinicopathological features, imaging features, therapeutic strategies and prognosis to provide helpful information in the diagnosis and treatment of PIM.
\end{abstract}

Case Description: A 56-year-old man presented with weakness and numbness in both legs for several years. Contrast-enhanced magnetic resonance imaging (MRI) of the spinal cord was performed. Based on the imaging examination, cavernous malformation with subacute hematoma was considered as the initial diagnosis. However, histopathological and immunohistochemical analyses confirmed the final diagnosis of PIM in the thoracic cord after surgical resection. The patient had no signs of recurrence or metastasis during a 17 -month follow-up.

Conclusions: MRI is the preferred method for the evaluation of PIM. PIM is characterized by a high signal on T1WI and a low signal on T2WI. It is difficult to make the differential diagnosis from cavernous malformation with hematoma before surgery due to its rarity. However, the symptom is not sudden but gradually worsened over a relatively long period in the PIM patients, which is an important difference from the cavernous malformation with hematoma. Therefore, PIM should receive diagnostic con $\neg$ sideration for an intramedullary lesion that is high signal on T1WI and low signal on T2WI in a patient with gradually worsened symptoms rather than sudden onset. It is of great importance for neurosurgeons and radiologists to recognize the characteristics of this disease, make the correct diagnosis in time and avoid delayed treatment.

Keywords: Intramedullary tumor; melanocytoma; thoracic cord; magnetic resonance imaging (MRI); case report

Submitted Oct 01, 2021. Accepted for publication Feb 17, 2022.

doi: $10.21037 /$ tcr-21-2132

View this article at: https://dx.doi.org/10.21037/tcr-21-2132

\section{Introduction}

Meningeal melanocytoma is a rare pigmented neoplasm of the central nervous system (CNS) first described in 1972 by Limas and Tio (1). Melanocytomas are derived from scattered melanocytes that are present in the leptomeninges. As for intraparenchymal melanocytomas, the melanocytes most probably originate from the Virchow-Robin spaces (2). In the spinal cord, most cases of meningeal melanocytomas are detected as intradural extramedullary lesions (3-5). The spine's primary intramedullary lesion is an extremely rare entity, with only 25 cases reported previously (2,4-18). Herein we report a case of primary intramedullary melanocytoma (PIM) in the thoracic cord in a 56-year-old man. In addition, the available literatures are reviewed. In this study, we presented a case of PIM and reviewed its clinicopathological features, imaging features, therapeutic strategies and prognosis to provide helpful information in the diagnosis and treatment of PIM. This study deepens our understanding of the clinical and imaging features of PIM. We present the following article in accordance with the CARE reporting checklist (available at https://tcr. 

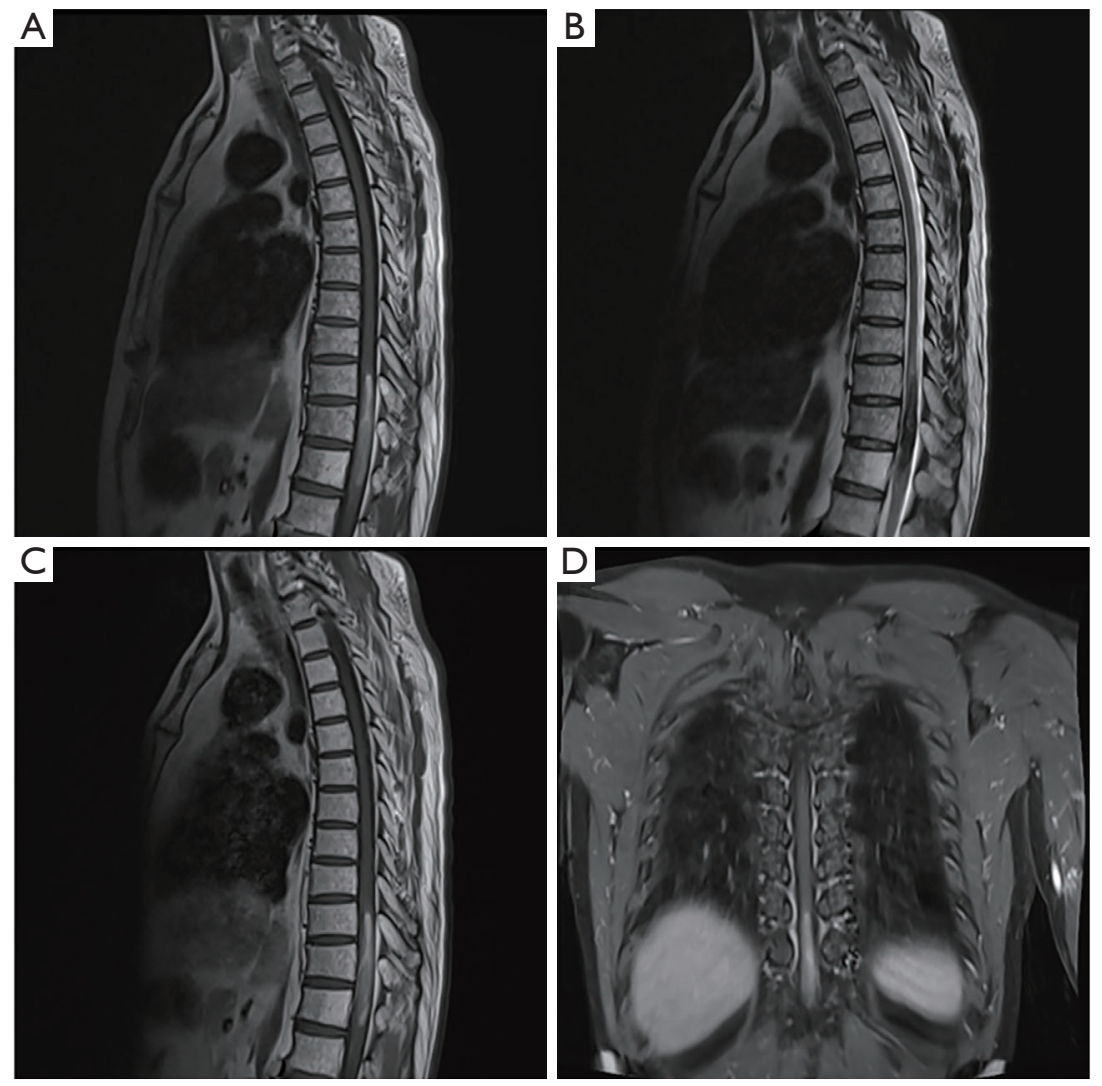

Figure 1 MRI revealed an intramedullary mass in the thoracic cord. The lesion occupied nearly $100 \%$ of the spinal canal at the level of T10-T12. The mass was T1 hyperintense (A) and T2 hypointense (B). On the contrast-enhanced T1-weighted image (C,D), the mass signal was similar to that on unenhanced T1WI (A). Therefore, there was an uncertain enhancement on the contrast-enhanced T1WI (C,D). T1WI, T1-weighted image; MRI, magnetic resonance imaging.

amegroups.com/article/view/10.21037/tcr-21-2132/rc).

\section{Case presentation}

\section{Clinical bistory}

A 56-year-old man presented with weakness and numbness in both legs for 3 years. The symptoms of weakness and numbness in both legs were aggravated for 10 months with walking unsteadily and sometimes falling over. Then, he was admitted to the department of neurosurgery in our hospital. The patient had a history of hypertension for 4 years and recently took Irbesartan to control blood pressure. Physical examinations on admission were as follows: soft neck with no resistance, grade IV muscle strength of both two lower extremities, grade $\mathrm{V}$ muscle strength of both two upper extremities, normal muscle tension, decreased pain, warmth, and touch sensation of both lower extremities below inguinal level, and negative bilateral pathological signs.

\section{Radiological examinations}

Magnetic resonance imaging (MRI) of the thoracic cord revealed an intramedullary mass, sized $8.0 \mathrm{~cm} \times 1.0 \mathrm{~cm}$ $\times 1.0 \mathrm{~cm}$, within the spinal canal. The lesion occupied nearly $100 \%$ of the spinal canal at the level of T10-T12 (Figure 1). The mass was T1 hyperintense (Figure $1 A$ ) and T2 hypointense (Figure 1B). On the contrast-enhanced T1weighted image (T1WI), the mass signal was similar to the mass signal on the unenhanced T1WI. Therefore, there was an uncertain enhancement on the contrast-enhanced T1WI (Figure 1C,1D). There was a small amount of edema above the mass in the spinal cord, as evidenced by $\mathrm{T} 2$ hyperintensity (Figure 1B). A cavernous malformation with subacute hematoma was considered as the initial diagnosis. 


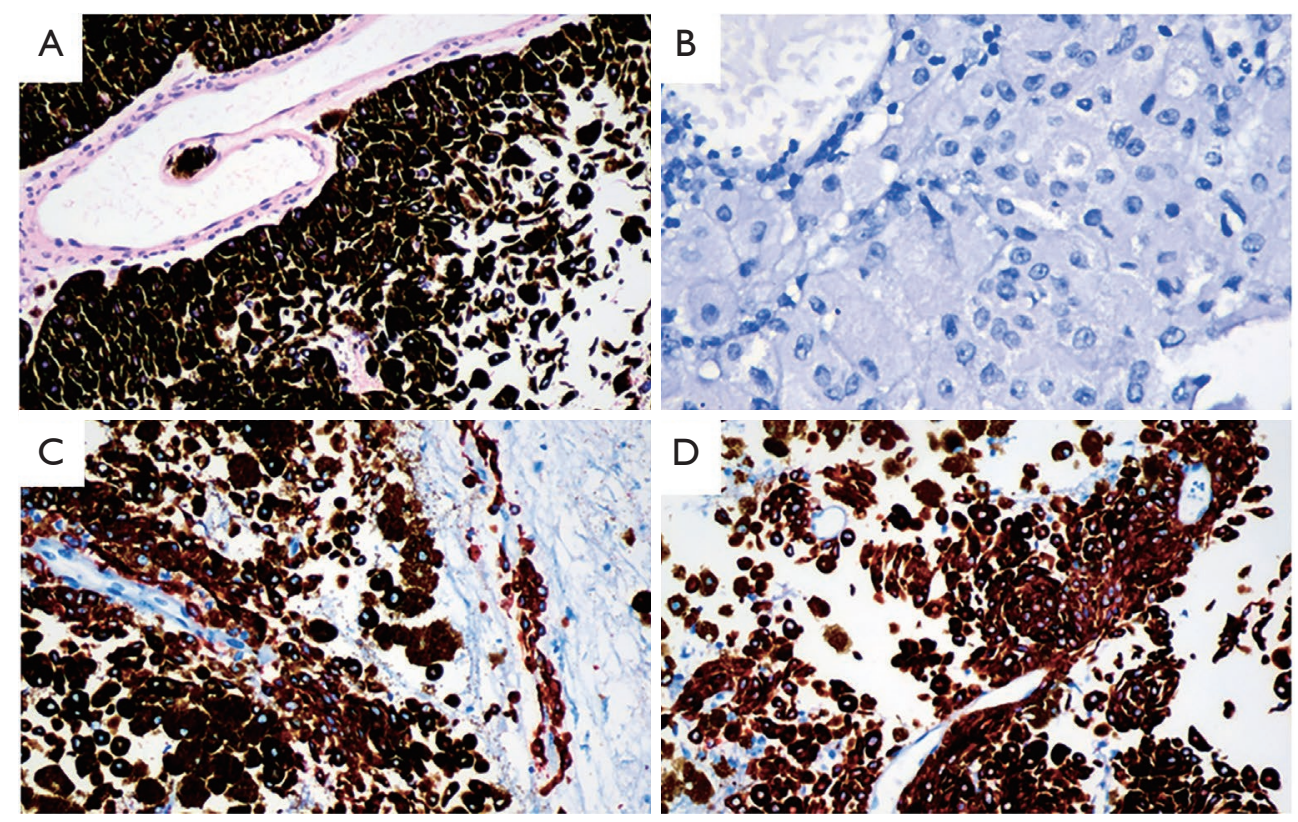

Figure 2 The patient's microscopic pathological and immunohistochemical results. (A) Histological staining showed that the tumor cells were arranged around the vascular axis, with poor adhesion, abundant melanin granules in the cytoplasm, large cell nuclei, and large nucleoli (HE, $\times 200)$. (B) After depigmentation, the large cell nuclei and nucleoli were more obvious $(\times 400)$. Immunohistochemical staining showed the tumor cells were positive for HMB45 (C) and Melan A (D) $(\times 200)$.

In addition, intramedullary melanoma ependymoma, astrocytoma, and schwannoma were considered the differential diagnosis.

\section{Surgical findings and pathological examination results}

A T10-T12 laminectomy was performed. The dura mater and arachnoid sheath were opened longitudinally. The spinal cord was opened along the posterior median sulcus to expose the intramedullary tumor. The tumor invaded deeply into the spinal cord and had an unclear boundary. And intraoperative frozen pathology did not determine the benign or malignant tumor. Thus, near-total resection of the tumor was performed.

Histological staining with hematoxylin-eosin showed that the tumor cells were arranged around the vascular axis, with poor adhesion, abundant melanin granules in the cytoplasm, large cell nuclei, and large nucleoli (Figure $2 A$ ). After depigmentation, large cell nuclei and nucleoli were more obvious (Figure 2B). Immunohistochemical staining showed the tumor cells were positive for HMB45 (Figure 2C), Melan A (Figure 2D), and negative for S-100, P53, BRAF. The Ki-67 proliferation index was low (5\%) in tumor cells. A diagnosis of PIM in the thoracic cord was finally determined.

\section{Post-operative course}

Postoperative muscle strength of the patient's right lower extremity was grade 2, and left lower extremity muscle strength was grade 1 . There was no muscle tension reduction in the extremities and no obvious abnormalities in the deep and shallow sensation. Moreover, bilateral Babinski signs were negative. Postoperative MRI indicated effusion in the operative region (Figure $3 A, 3 B$ ). The patient was discharged six days later after surgery. After a followup of 17 months, the patient had no signs of recurrence or metastasis. The timeline of the diagnosis, treatment, development, and prognosis is shown in Figure 4.

All procedures performed in this study in accordance with the ethical standards of the institutional and/or national research committee(s) and with the Helsinki Declaration (as revised in 2013). Written informed consent was obtained from the patient for publication of this case report and accompanying images. A copy of the written consent is available for review by the editorial office of this journal. The study protocols were approved by the ethics committee of our hospital (No. 2021-0013). 

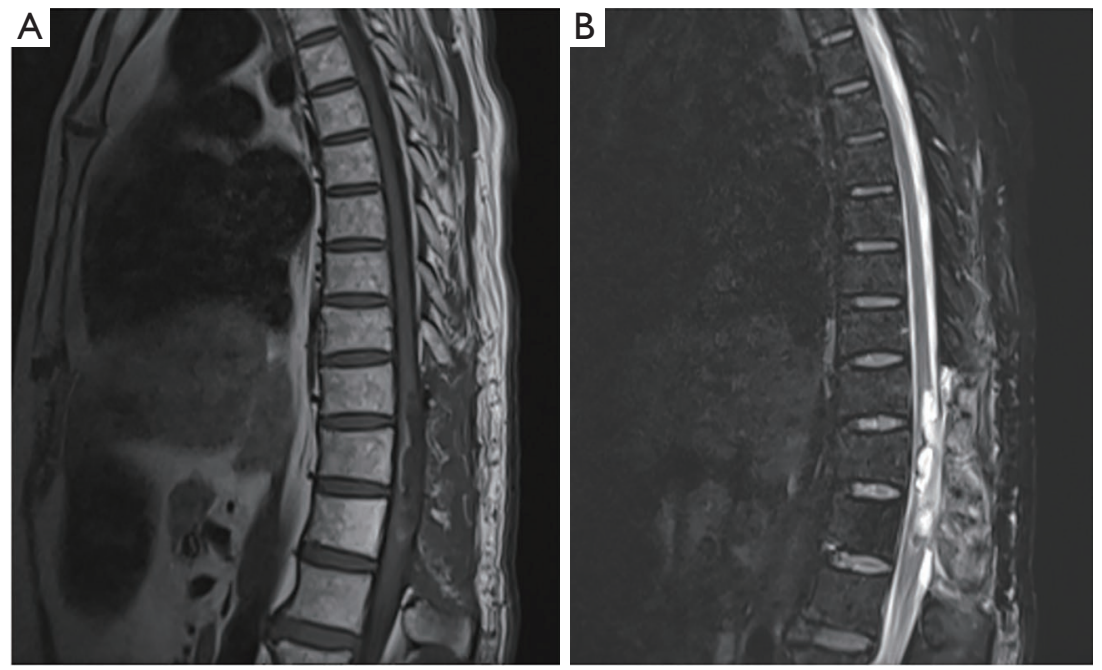

Figure 3 Postoperative MRI indicated effusion in the resection region. MRI, magnetic resonance imaging.

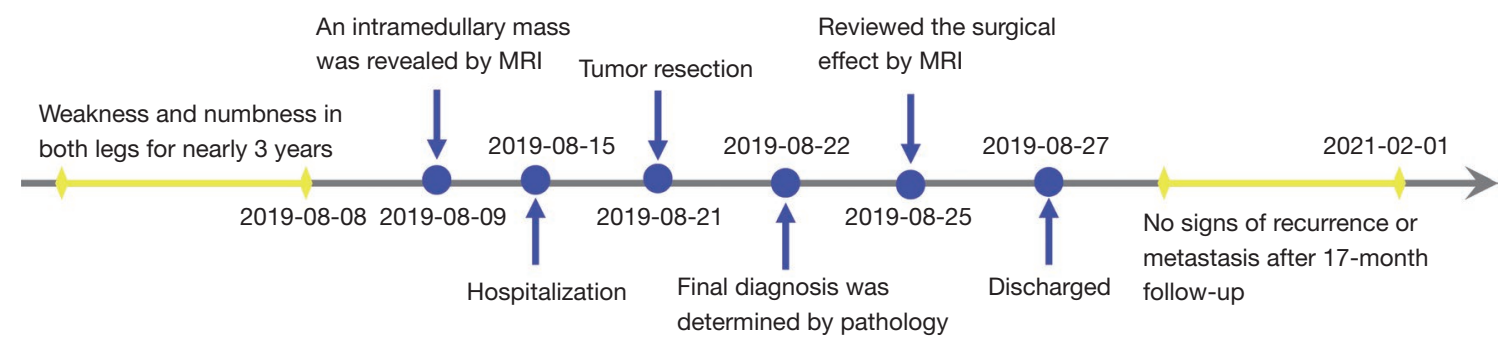

Figure 4 The timeline of management.

\section{Discussion}

Primary CNS melanocytoma is a rare disease that originates from melanocytes located in the leptomeninges (19) and is considered a benign neoplastic lesion. Tumors of the same origin range from well-differentiated melanocytoma to malignant melanoma, and their prognosis is quite different. According to the classification of tumors of the CNS by the World Health Organization (WHO) (20), primary melanocytic lesions are classified into four categories, including melanocytosis, melanocytoma, malignant melanoma, and meningeal melanomatosis. Melanocytoma is more common in the spinal cord than in the brain. In the spinal cord, primary melanocytoma is more likely to occur in the cervical and thoracic regions, usually presented as an extramedullary intradural lesion (21). However, primary melanocytoma is rarely presented as an intramedullary region, with only 25 cases reported previously (Table 1) (2,4-18). In this study, we present a comprehensive description of PIM, including its clinical and radiological features.

The clinical symptoms of melanocytoma patients are generally caused by the tumor's compression, including lower limb movement disorders, numbness, paresthesia, hypoesthesia, abnormal stool, and urine, etc., which are related to the location of the compression and the size of the tumor (22). Root pain is less common in the intramedullary melanocytoma than the extramedullary epidural melanocytoma.

Radiological examinations can show the location, extent, interior features, and the tumor's compression on the spinal cord. MRI is the preferred method for the evaluation of spinal cord tumors. PIM is characterized by a high signal on T1WI and a low signal on T2WI $(23,24)$, which may be related to the paramagnetic free radicals generated by a large amount of melanin and hemorrhage in the melanocytoma (25). By contrast, a non-melanotic tumor is low signal on T1WI and high signal on T2WI, which helps 
Table 1 Summary of primary intramedullary melanocytoma cases in the spinal cord reported in the literatures

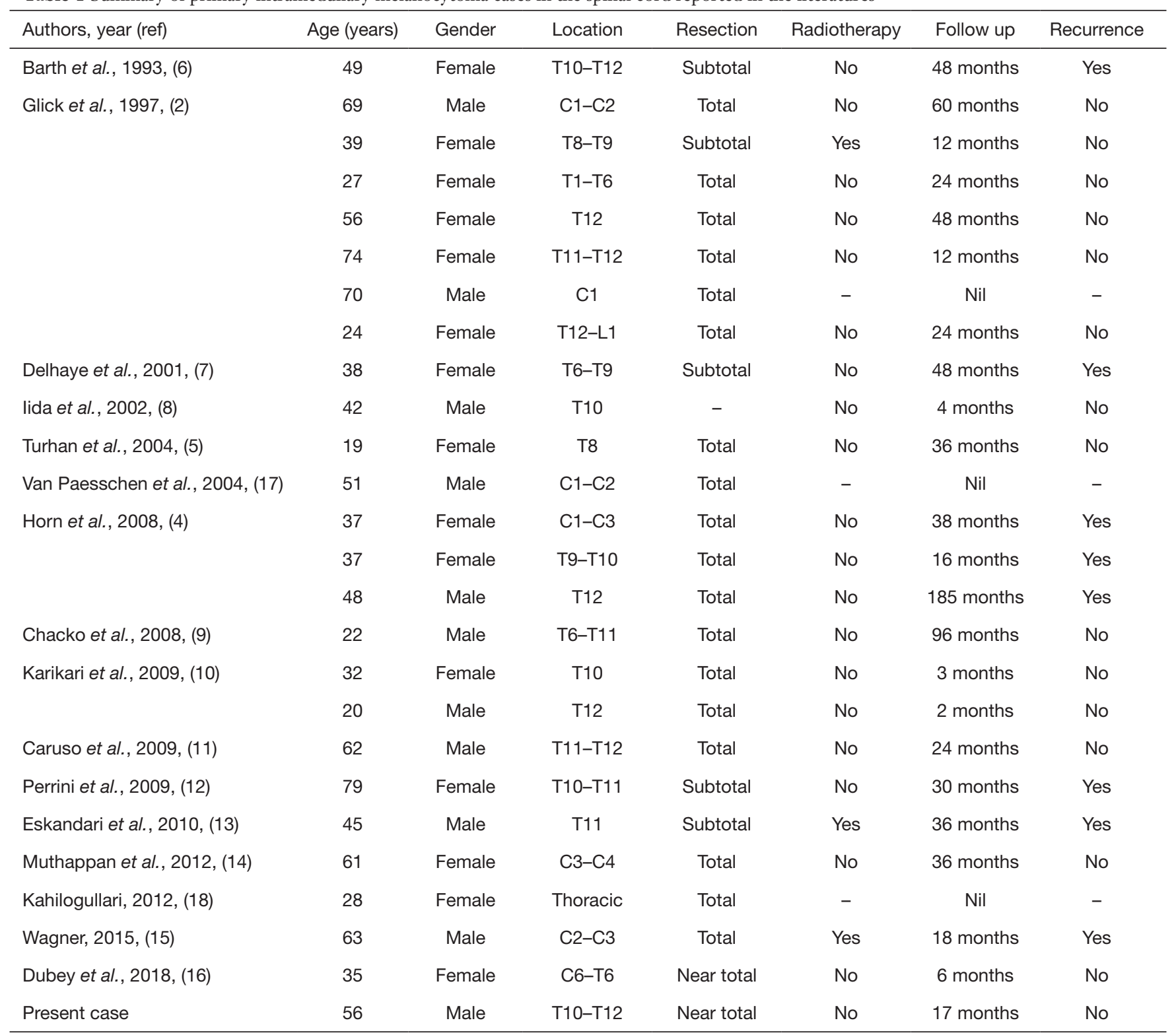

differentiate melanotic from non-melanotic lesions, such as ependymoma, astrocytoma, and schwannoma. Despite this imaging feature of melanocytoma, it is difficult to distinguish from subacute hematoma caused by hemorrhage of intramedullary cavernous malformation. In the present case, a cavernous malformation with subacute hematoma was initially considered in the diagnosis. However, the hemorrhage often appears as a sudden onset, and the imaging manifestations and symptoms can be gradually recovered with the absorption of the hematoma. Although the intramedullary melanocytoma reveals specific imaging characteristics, the final diagnosis depends on postoperative pathology and immunohistochemistry.

According to the previous case reports (Table 1), surgical resection is the primary treatment for melanocytoma $(2,4-18)$. Although melanocytoma is a benign tumor, it may recur after complete resection, and it may also invade surrounding tissues after surgery (26). This tumor's high recurrence risk and aggressive behavior (13) suggest the need for continuous close follow-up. The tumor should be resected entirely as far as possible, and adjuvant radiotherapy is recommended for patients with total or 
partial resection, which can prevent tumor recurrence and significantly improve prognosis $(22,27)$.

In summary, MRI is the preferred method for the evaluation of PIM. PIM is characterized by high signal on T1WI and low signal on T2WI. This imaging feature can help differentiate melanotic from non-melanotic lesions, such as ependymoma, astrocytoma, and schwannoma. Due to its rarity, it is difficult to make the differential diagnosis from cavernous malformation with hematoma before surgery. However, the symptoms are not sudden but gradually worsened over a relatively long period in the PIM patients, which is an essential difference from the cavernous malformation with hematoma. Therefore, PIM should receive diagnostic consideration for an intramedullary lesion that is high signal on T1WI and low signal on T2WI in a patient with gradually worsened symptoms rather than sudden onset. It is of great importance for neurosurgeons and radiologists to recognize the characteristics of this disease, make the correct diagnosis in time and avoid delayed treatment.

\section{Acknowledgments}

The authors would like to thank the efforts of Yeerfan Jiaerken for revising the language.

Funding: This research was supported by Zhejiang Provincial Natural Science Foundation of China (LY21H180003).

\section{Footnote}

Reporting Checklist: The authors have completed the CARE reporting checklist. Available at https://tcr.amegroups.com/ article/view/10.21037/tcr-21-2132/rc

Conflicts of Interest: All authors have completed the ICMJE uniform disclosure form (available at https://tcr.amegroups. com/article/view/10.21037/tcr-21-2132/coif). The authors have no conflicts of interest to declare.

Ethical Statement: The authors are accountable for all aspects of the work in ensuring that questions related to the accuracy or integrity of any part of the work are appropriately investigated and resolved. All procedures performed in this study in accordance with the ethical standards of the institutional and/or national research committee(s) and with the Helsinki Declaration (as revised in 2013). Written informed consent was obtained from the patient for publication of this case report and accompanying images. A copy of the written consent is available for review by the editorial office of this journal. The study protocols were approved by the ethics committee of our hospital (No. 2021-0013).

Open Access Statement: This is an Open Access article distributed in accordance with the Creative Commons Attribution-NonCommercial-NoDerivs 4.0 International License (CC BY-NC-ND 4.0), which permits the noncommercial replication and distribution of the article with the strict proviso that no changes or edits are made and the original work is properly cited (including links to both the formal publication through the relevant DOI and the license). See: https://creativecommons.org/licenses/by-nc-nd/4.0/.

\section{References}

1. Limas C, Tio FO. Meningeal melanocytoma ("melanotic meningioma"). Its melanocytic origin as revealed by electron microscopy. Cancer 1972;30:1286-94.

2. Glick R, Baker C, Husain S, et al. Primary melanocytomas of the spinal cord: a report of seven cases. Clin Neuropathol 1997;16:127-32.

3. Brat DJ, Giannini C, Scheithauer BW, et al. Primary melanocytic neoplasms of the central nervous systems. Am J Surg Pathol 1999;23:745-54.

4. Horn EM, Nakaji P, Coons SW, et al. Surgical treatment for intramedullary spinal cord melanocytomas. J Neurosurg Spine 2008;9:48-54.

5. Turhan T, Oner K, Yurtseven T, et al. Spinal meningeal melanocytoma. Report of two cases and review of the literature. J Neurosurg 2004;100:287-90.

6. Barth A, Pizzolato GP, Berney J. Intramedullary meningeal melanocytoma. Neurochirurgie 1993;39:188-94.

7. Delhaye M, Menei P, Rousselet MC, et al. A case of intramedullary primary melanocytic tumor: meningeal melanocytoma or malignant melanoma? Neurochirurgie 2001;47:133-6.

8. Iida M, Llena JF, Suarez MA, et al. Two cases of spinal meningeal melanocytoma. Brain Tumor Pathol 2002;19:41-5.

9. Chacko G, Rajshekhar V. Thoracic intramedullary melanocytoma with long-term follow-up. J Neurosurg Spine 2008;9:589-92.

10. Karikari IO, Powers CJ, Bagley CA, et al. Primary intramedullary melanocytoma of the spinal cord: case report. Neurosurgery 2009;64:E777-8; discussion E778.

11. Caruso R, Marrocco L, Wierzbicki V, et al. Intramedullary 
melanocytoma: case report and review of literature.

Tumori 2009;95:389-93.

12. Perrini P, Caniglia M, Pieroni M, et al. Malignant transformation of intramedullary melanocytoma: case report. Neurosurgery 2010;67:E867-9; discussion E869.

13. Eskandari R, Schmidt MH. Intramedullary spinal melanocytoma. Rare Tumors 2010;2:e24.

14. Muthappan M, Muthu T, Hussain Z, et al. Cervical intramedullary melanocytoma: a case report and review of literature. J Clin Neurosci 2012;19:1450-3.

15. Wagner F, Berezowska S, Wiest R, et al. Primary intramedullary melanocytoma in the cervical spinal cord: Case report and literature review. Radiol Case Rep 2015;10:1010.

16. Dubey A, Kataria R, Sardana VR. Intramedullary Melanocytoma of the Cervicothoracic Cord: Case Report and Review of Literature. Asian J Neurosurg 2018;13:478-81.

17. Paesschen RV, Calenbergh FV, Demaerel P, et al. Intramedullary melanocytoma associated with syringobulbia. Acta Neurologica Belgica 2004;104:132-3.

18. Kahilogulları G, Bozkurt M, Cakıroglu E, et al. Primary intramedullary melanocytoma: a case report. World Spinal Column Journal 2012;3:64-6.

19. Liubinas SV, Maartens N, Drummond KJ. Primary melanocytic neoplasms of the central nervous system. J Clin Neurosci 2010;17:1227-32.

Cite this article as: Wang C, Shao X, Zou Y. Primary intramedullary melanocytoma in the thoracic cord: a case report and literature review. Transl Cancer Res 2022;11(4):928-934. doi: $10.21037 /$ tcr-21-2132
20. Brat DJ, Parisi JE, Kleinschmidt-DeMasters BK, et al. Surgical neuropathology update: a review of changes introduced by the WHO classification of tumours of the central nervous system, 4th edition. Arch Pathol Lab Med 2008;132:993-1007.

21. Reutov AA, Ryzhova MV, Kushel YV. Intramedullary melanocytoma: a clinical case report and literature review. Zh Vopr Neirokhir Im N N Burdenko 2016;80:75-80.

22. Hoffmann M, Koelsche C, Seiz-Rosenhagen M, et al. The GNAQ in the haystack: intramedullary meningeal melanocytoma of intermediate grade at T9-10 in a 58-year-old woman. J Neurosurg 2016;125:53-6.

23. Gupta PK, Misra S, Verma R, et al. Primary intradural cervical spine melanocytoma: A rare tumor and review of literature. Neurol India 2017;65:653-7.

24. Smith AB, Rushing EJ, Smirniotopoulos JG. Pigmented lesions of the central nervous system: radiologic-pathologic correlation. Radiographics 2009;29:1503-24.

25. Hu L, Wang C. Intramedullary melanotic schwannoma of the cervical spine: A case report and literature review. Mol Clin Oncol 2018;8:567-70.

26. Rades D, Schild SE, Tatagiba M, et al. Therapy of meningeal melanocytomas. Cancer 2004;100:2442-7.

27. Rades D, Tatagiba M, Brandis A, et al. The value of radiotherapy in treatment of meningeal melanocytoma. Strahlenther Onkol 2002;178:336-42. 